\title{
SEROPREVALENCIA DE VIH, HEPATITIS B, HEPATITIS C, CHAGAS Y SIFILIS EN DONANTES DE BANCOS DE SANGRE DE CÚCUTA (COLOMBIA) 1.998 - 1.999
}

\author{
AUTORES \\ GLORIA ESPERANZA ZAMBRANO \\ PLATA. \\ Profesora Asistente Universidad \\ Francis-co de Paula Santander \\ JAVIER CORTEZ \\ Profesor Cátedra Universidad Francisco \\ De Paula Santander
}

\section{RESUMEN}

Para determinar la seroprevalencia de enfermedades transmisibles en donantes de los bancos sangre de la ciudad de Cúcuta (N.S.), se realizó un estudio descriptivo retrospectivo, para lo cual se revisaron los reportes de las pruebas de tamizaje realizadas a los donantes durante 1.998 -1.999. Se definieron como variables del estudio: unidades de sangre reactivas para anticuerpos contra el virus de inmunodeficiencia humana $(\mathrm{VIH})$, virus de la hepatitis $\mathrm{C}$ (VHC), Tryponosoma cruzi, Treponema pallidum y antígenos de superficie del virus de la hepatitis $B$ (VHB). Además se incluyeron otras variables para describir la población seropositiva como son: edad, sexo, grupo sanguíneo y procedencia. Se encontró una seroprevalencia de $6.29 \%$ de enfermedades transmisi-bles en los donantes de sangre. Del total de unidades de sangre analizadas el $0.28 \%$ resulto reactiva para el VIH, el $1.21 \%$ para el HVB, $0.54 \%$ para el VHC, $2.92 \%$ para Chagas, $1.71 \%$ para sífilis y un $0.37 \%$ presentan dos o más pruebas reactiva.

\section{INTRODUCCION}

EI VIH, la hepatitis $B$, la hepatitis $C$, la enfermedad de Chagas y la Sífilis son enfermedades con altas tasas de prevalencia especialmente en países subdesarrollados. El curso crónico y/o letal al igual que la facilidad con que se trasmiten estas enfermedades las convierten en un verdadero problema de salud Pública.

Con el fin de prevenir la transmisión de estas enfermedades en los receptores de sangre, se implementaron las pruebas de tamizaje a los donantes de sangres, mediante el decreto 559 de 1.991 y 571 de 1.993 expedidos por el ministerio de Salud de Colombia, se estableció como pruebas obligatorias en los bancos de sangre: anticuerpos anti $\mathrm{VIH}$, tipo 1 y 2 , antígenos de superficie de hepatitis $B$, anticuerpos anti virus de la hepatitis $C$ y anticuerpo anti treponema pallidum. En 1.995 para la detección de la enfermedad de Chagas se adopto la prueba de anticuerpos anti tripanosoma cruzi.

En la ciudad de Cúcuta funcionan en la actualidad 4 bancos de sangre (Clínica San José de Cúcuta, SANALAC, Instituto de seguros sociales y Hospital Erasmo Meóz) que atienden población proveniente de todo el departamento Norte de Santander y Arauca. Esta población proviene de áreas que tradicionalmente han tenido una prevalencia elevada de enfermedades transmi- sibles; de acuerdo al reporte de los tamizajes realizados en los bancos de sangre de Colombia, Norte de Santander presenta una seroprevalencia para VIH, VHB, Chagas y Sífilis superior al promedio nacional (Beltrán, 1997, 137-142).

\section{MATERIALES Y METODOS}

Se realizó un estudio retrospectivo. Para esto el grupo investigador revisó la totalidad de los registros existentes en los cuatro bancos, sobre el resultado del tamizaje realizado a las unidades de sangre.

Los bancos de sangre realizan pruebas de ELISA (inmunoensayo) para la detección de: anticuerpos frente al $\mathrm{VIH}$, antígenos de superficie para hepatitis $\mathrm{B}$, anticuerpos anti VHC y anticuerpos anti Triponosoma cruzi (Enfermedad de Chagas). Para la detección de anticuerpos anti Treponema pallidum (Sífilis) se realiza la prueba de UMELISA (inmunoensayo) (VDRL).

Se definieron como variables del estudio: unidades de sangre reactivas para anticuerpos contra el virus de inmunodeficiencia humana (VIH), virus de la hepatitis $\mathrm{C}(\mathrm{VHC})$,

Tryponosoma cruzi, Treponema pallidum y antígenos de superficie del virus de la hepatitis $B$ (VHB). Ade-más se incluyeron otras variables para describir la población seropositiva como son: edad, sexo, grupo sanguíneo y procedencia. 
La información obtenida fue sometida a un análisis descriptivo, con lo cual se puede tener una aproximación de la magnitud de la prevalencia del SIDA, hepatitis $B$, hepatitis $C$, chagas y sífilis, y características demográficas de la población con serologías positivas o reactivas. Esta información permitirá reforzar los programas de promoción prevención y control de las enfermedades transmisibles.

\section{RESULTADOS}

Durante el período comprendido entre 1.998 y 1.999 , los cuatro bancos de sangre de la ciudad de Cúcuta realizaron pruebas de tamizaje a 17.895 unidades de sangre donadas, encontrándose en 1.125 (6.29\%) de estas unidades seropositividad; dentro de este grupo se destacan 67 pacientes $(0,37 \%)$ con más de una prueba positiva o reactiva (Cuadro No.1). La serología positiva para la enfermedad de Chagas presentó la mayor prevalencia $(2.92 \%)$, seguido por la prueba de selección de sífilis: VDRL reactivo (1.71\%) y VHB (1.21\%). En menor porcentaje se presentó serologías positivas para VHC (0.54\%) y para VIH (0.28\%). (Cuadro No. 2 y Gráfica No.1)

\section{CUADRO No. 1 SEROPREVALENCIA EN} DONANTES DE SANGRE CUCUTA 1.998-1.9995

\begin{tabular}{lcccc} 
RESULTADOS & Frecuencia & F\% & TOTAL & $\%$ \\
Una prueba positiva & 1058 & 5,912 & 1125 & 6,29 \\
Dos prubas o más positivas & 67 & 0,374 & & \\
Negativo & 16770 & 93,71 & 16770 & 93,7 \\
TOTAL & 17895 & 100 & 17895 & 100 \\
\multicolumn{4}{c}{ Fuente: elaboración propia } \\
\end{tabular}

CUADRO No.2 SEROPREVALENCIA EN DONANTES DE SANGRE CUCUTA 1.998-1.995

\begin{tabular}{|c|c|c|}
\hline PRUEBA & FRECUENCIA & $\%$ \\
\hline Chagas & 523 & 2,92 \\
VDRL & 306 & 1,71 \\
VHC & 96 & 0,54 \\
VHB & 216 & 1,21 \\
HIV & 51 & 0,28 \\
\hline
\end{tabular}

\section{GRAFICA No. 1 SEROPREVALENACIA ESPECIFICA EN DONANTES DE SANGRE CUCUTA 1.998-1.999}

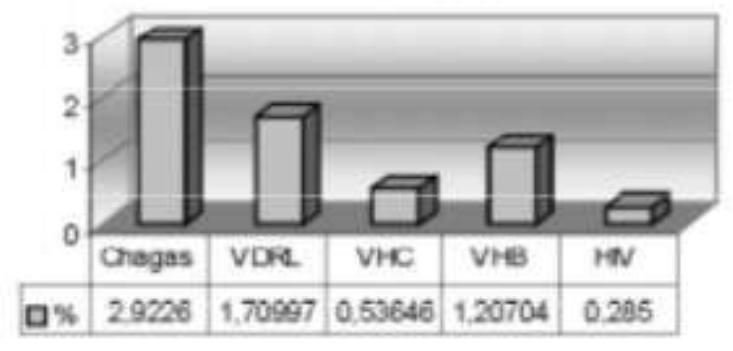

El $85 \%$ de los donantes seropositivos son adultos en edad productiva (17-46años) y el menor porcentaje se observa en los donantes mayores de 60 años El grupo de edad más afectado es el de 27 a 36 años $(39,8 \%)$, los cuales presentan una alta positividad para Chagas (17\%), seguido de VDRL(11\%) y VHB (7,5\%). En segundo lugar se encuentra el grupo de edad entre 37 y 46 años $(25,4 \%)$, predominando en este grupo igual que el anterior el Chagas (14\%), el VDRL (4\%) y VHB (4\%) en su orden. En el grupo de 17 a 26 años se observa un predominio de Sífilis y VHB (6\% c/u) seguido de Chagas (4\%) (Gráfica No. 2).

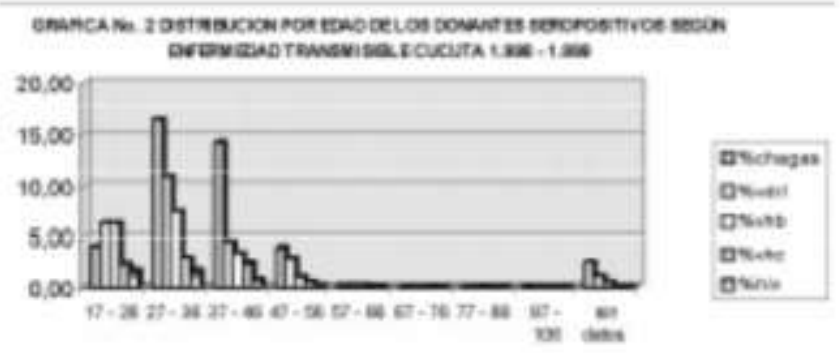

Fuente: elaboración propia

La prevalencia de las enfermedades transmisibles es mucho mayor en los hombres en relación con las mujeres. Se encontró en general que por cada 12 hombres con serología positiva hay una mujer. Especificando por pruebas por cada 12 hombres con serología positiva para Chagas y VIH hay una mujer. La mayor diferencia se observó en los donantes con mas de una prueba positiva, donde por cada 21 hombres hay una mujer y la menor diferencia se presento en los pacientes con serología positiva para VHC donde por cada 9 hombres hay una mujer (Cuadro No.3) 


\section{CUADRO No. 3 PREVALENCIA POR SEXO Y RAZON DE MASCULINIDAD DE LOS DONATES DE SANGRE SEROPOSITIVOS CÚCUTA 1.998 - 1.999}

\begin{tabular}{|c|c|c|c|c|c|}
\hline & \multicolumn{2}{|c|}{ Mascuutio } & \multicolumn{2}{|c|}{ FEMEIIHO } & \multirow{2}{*}{$\begin{array}{l}\text { RAzOKi } \\
\text { MFF }\end{array}$} \\
\hline & FECUEIOA & 8 & Fbouftios & 8 & \\
\hline Crenpe & 433 & 2.419 & 36 & 0301 & 12 \\
\hline coti. & 201 & $(3,458$ & 18 & Q.100 & 74 \\
\hline WE & 87 & Q334 & 5 & 0,044 & 8 \\
\hline Whe & 391 & 0.855 & 16 & 0,100 & 8 \\
\hline the & 40 & 0.223 & 4 & 0.022 & 10 \\
\hline Morios & ज्ञ & 0357 & 3 & 6010 & 21 \\
\hline Tot4 & 1056 & 5,280 & ${ }^{\circ}$ & 0.402 & 22 \\
\hline
\end{tabular}

La distribución de los grupos sanguíneos en los donantes de sangre con serología positiva o reactiva para $\mathrm{VIH}, \mathrm{VHB}, \mathrm{VHC}$, enfermedades de Chagas y Sífilis es muy similar (Gráfica No. 3). Mas de la mitad de los donantes son O positivo (54.4\%), seguido por el grupo A positivo (33.16\%). Para el factor $\mathrm{RH}$ negativo el mayor porcentaje esta dado por el grupo $\mathrm{O}$ negativo. Se encontró una baja frecuencia del grupo $B$ negativo $(0.08 \%)$ y ningún donante seropositivo con el grupo $A B$ negativo.

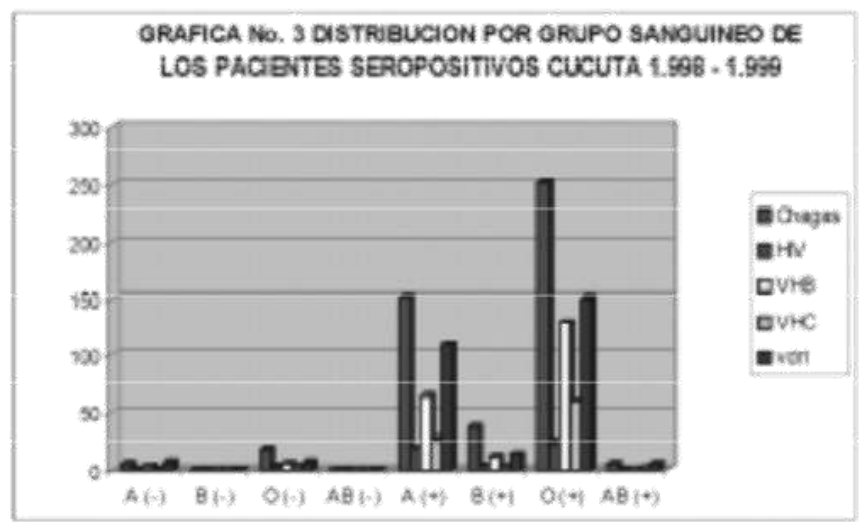

El $91 \%$ de los donantes seropositivos provienen de la zona urbana, en un $5 \%$ de los donantes se desconoce su procedencia (Gráfica No. 4). Tres Cuartas partes de estos pacientes provienen de la ciudad de Cúcuta (76\%), en orden le sigue el municipio de Los Patios (4,71\%), Villa del Rosario (4,44\%), El Zulia (2,22\%) y Tibú (1,78\%). Por el carácter fronterizo de la ciudad de Cúcuta el 1,33\% de los donantes seropositivos provienen de la república de Venezuela (Cuadro No. 4). Con relación a los donantes seropositivos provenientes del municipio de Cúcuta, se encontró que la mayor proporción proviene de la comuna $8(13,5 \%), 7(13,3 \%), 9(9,6 \%)$ y $6(8.85 \%)$.
El menor porcentaje se observa en la comuna 12 $(1,2 \%)$ (Cuadro No. 5 y Gráfica No. 5)

CUADRO No. 4 PROCEDENCIA POR GRAFICA NO. 4 PROCEDENCIA DE LOS DONANTES SEROPOSITIVOS CUCUTA $1.998-1.999$

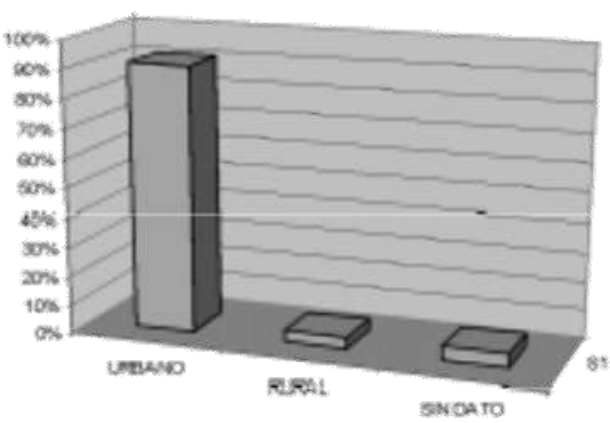

\section{MUNCIPIO DE LOS DONANTES SEROPOSITIVOS CÚCUTA}

$1.998-1.999$

\begin{tabular}{|c|c|c|}
\hline POCEDEHCIA & TOTAL & 8 \\
\hline BARRAIICABERME IA & 1 & 0,09 \\
\hline BUCARAMANGA & 3 & 0,27 \\
\hline CASANARE & T & 0,09 \\
\hline OHINACOIA & 3 & 0,27 \\
\hline CORUENGIOH & 1 & 0.09 \\
\hline CORIENO & 2 & 0,18 \\
\hline CUCUTA & 859 & 76,36 \\
\hline DURANIIA & 1 & 0,09 \\
\hline EL CARMEM & 1 & 0,09 \\
\hline EL TARRA & 5 & 0,44 \\
\hline GRAMALOTE & 2 & 0,18 \\
\hline LA,BATECA & 1 & 0.09 \\
\hline LA DOHA NARAA & 1 & 0,09 \\
\hline LA GABARRA & 3 & 0,27 \\
\hline OCANA & 3 & 0,27 \\
\hline PAMPLONA & 1 & 0,09 \\
\hline LOS PATIOS & 53 & 4,71 \\
\hline PUERTO SAMIIAMDER & 12 & 1.07 \\
\hline RACONNALLA & 1 & 0.09 \\
\hline SALAZAR & 2 & 0.18 \\
\hline SANH CAYETANIO & $T$ & 0.09 \\
\hline SAN FAUSTINO & I & 0,09 \\
\hline SARAVETHA & 2 & 0,18 \\
\hline SARDIIATA & 5 & 0,44 \\
\hline nBU & 20 & 1,78 \\
\hline TOLEDO & 3 & 0,27 \\
\hline MLLA ROSARIO & 50 & 4,44 \\
\hline ZUUA & 25 & 2,22 \\
\hline VENEZUELA & 15 & 1,33 \\
\hline SITIDATO & 47 & 4,18 \\
\hline TOTAL & 1.125 & 100,00 \\
\hline
\end{tabular}


CUADRO No. 5 DISTRIBUCION POR COMUNASDE LOS DOANATES SEROPOSITIVOS PROCEDENTES DEL MUNICIPIO DE CUCUTA

\begin{tabular}{|c|c|c|}
\hline Co्millu & Fifculinats & 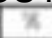 \\
\hline 1 & 29 & $\sqrt{3,30}$ \\
\hline 2 & 19 & 2.21 \\
\hline 3 & 42 & $4,0 \mathrm{k}$ \\
\hline 4 & 25 & 4,31 \\
\hline 5 & 3 & 2,7 \\
\hline 6 & 76 & E,ES \\
\hline 7 & 114 & 13.3. \\
\hline in & 117 & 13.6 \\
\hline 9 & 82 & 9,56 \\
\hline 10 & 46 & 3.16 \\
\hline 11 & 4 & 0,4 \\
\hline 12 & 1 & 0,12 \\
\hline \$ittokro & 260 & 30,3 \\
\hline $101 \times 1$ & 854 & 1700 \\
\hline
\end{tabular}

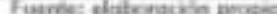

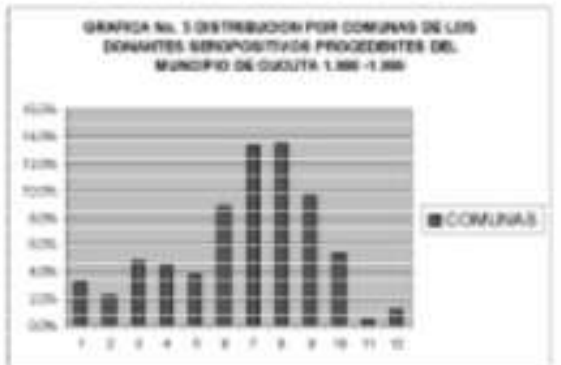

$1.998-1.999$

DISCUSION

Los donantes de sangre generalmente son asintomáticos y de bajo riesgo por lo tanto la seroprevalencia encontrada en este grupo es similar al de la población general (Beltrán, 1999). Las pruebas de tamizaje detectaron un $6.29 \%$ de unidades reactivas para HIV, VHB, VHC, enfermedad de Chagas o Sífilis, esta prevalencia es superior a la encontrada en Colombia (6.08\%) por Beltrán y colaboradores en 1.995 .

Los resultados evidencian que 2.8 de cada 1000 unidades de sangre resultan reactivas para $\mathrm{VIH}$, obsevandose un incremento importante, al encontrado en Norte de Santander en 1.995, que era de 2 por cada 1.000 unidades de sangre. Igualmente, la prevalencia registrada para el HIV en el actual estudio, es superior a la reportada en 1.994 y 1.995 en donantes de sangre en Argentina (0.2\%), Bolivia (0.03\%), Costa Rica (0.1\%), Ecuador $(0.18 \%)$, El Salvador (0.15\%), Nicaragua $(0.11 \%)$, Panamá $(0.12 \%)$ y Paraguay $(0.05 \%)$. Sólo Honduras $(0.5 \%)$ y Venezuela $(0.38 \%)$ informaron seroprevalencias superiores a la encontrada en la ciudad de Cúcuta (Organización Panamericana de La Salud, 1997).

Comparando la seroprevalencia reportada en 1.995 para VHB (3.9\%) y enfermedad de Chagas (3.5\%) con la reportada en 1.998-1.999, se observa una importante reducción (VHB:1.21\% y enfermedad de Chagas:2.92\%).

Dentro de estas enfermedades transmisibles la seroprevalencia más baja se observa en el VHC, que es la única que presenta una tasa inferior al promedio nacional y de los países latinoamericanos.

Es alarmante el número de donantes con dos pruebas reactivas, casi 3.7 de cada 1000 unidades de sangre analizadas presentan esta situación. Esto se constituye en un verdadero problema tanto para los contactos como para los mismos pacientes, quienes tienen una mayor susceptibilidad a presentar compli- caciones asociadas a estas patologías en forma precoz.

En los bancos de sangre estudiados, se encontró que la mayoría de las pacientes seropositivos son hombres principalmente entre 17 y 46 años. Esto se presenta, por que generalmente los donantes se encuentran en edad productiva y además es escaso el número de mujeres que donan debido a mitos que existen en la región alrededor de la donación de sangre por parte del genero femenino.

Como era de esperarse, la mayoría de los donantes seropositivos provienen de la zona urbana. Por el fácil acceso que tienen los donantes de los bancos de sangre que se localizan en la ciudad de Cúcuta, más de dos terceras partes proceden de Cúcuta y su área metropolitana (municipio de Villa del Rosario, Los Patios y Zulia). Para los casos procedentes del casco urbano del municipio de Cúcuta, se encontró una mayor seroprevalencia en las comunas ubicadas en la ciudadela Juan Atalaya, que corresponde a los estratos socioeconómicos más bajos.

Las características étnicas de la Población Cúcuteña y Nortesantandereana explican el predominio del grupo sanguíneo $O$ positivo en los donantes con serologias reactivas. Como es bien sabido este grupo sanguíneos característicos de la raza indígena y la población de esta región tiene descendencia indígena.

La alta prevalencia de infecciones transmisibles en donantes de origen 
urbano, se puede explicar en que la mayoría de los donantes residen en la zona urbana, pero a su vez, provienen de áreas rurales, que a raíz del incremento en la migración hacia las ciudades de los habitantes de zonas tradicionalmente endémicas para estas enfermedades, han originado un cambio en la distribución geográfica de las enfermedades transmisibles.

\section{CONCLUSIONES}

Las pruebas de tamizaje realizadas en los bancos de sangre, permitieron poner en evidencia un importante problema de salud pública en Cúcuta y Norte de Santander, como es la alta seroprevalencia de HIV, VHB, VHC enfermedad de Chagas y Sífilis.

Las pruebas de tamizaje permiten detectar enfermedades infecciosas, evitando de esta forma la transmisión de las mismas a los receptores de sangre. Sin embargo todavía no se puede hablar de transfusiones de riesgo cero debido al período de ventana inmunológica.

Los bancos de sangre al realizar estas pruebas de tamizaje no solo reducen la transmisión de estas enfermedades, sino que se convierten en unidades de vigilancia epidemiológica, que permiten detectar pacientes con serologías reactivas o positivas, posteriormente estos casos se deben confirmar y hacer el seguimiento a los pacientes sospechosos y/o confirmados.

Infortunadamente en esta región muchos pacientes se pierden sin poder confirmase el diagnostico y menos aún realizar un seguimiento. Esto dificulta conocer la prevalencia de infecciones activas, historia natural de estas enfermedades, factores de riesgo asociados específicos de estas comunidades, que permitan identificar medidas preventivas y de tratamiento oportuno

\section{BIBLIOGRAFIA}

BELTRÁN, M. et al. Tamizaje de enfermedades infeccio-sas en bancos de sangre, Colombia, 1995. En: Biomédica. No.17 (1997); p. 137-142

AYALA M. y LARA, J. Frecuencia de grupos san-guíneos y factor $R h$ en donantes de sangre. En: Biomédica. No. 19 (1996); p. 39-44

Prevención de la transmisión de Hepatitis C. Con-senso Colombiano de Hepatitis C. (oct.1999)

DÍAZ CASTRILLON, J. y ARROYAVE VELEZ, A. Estudios sobre Hepatitis B en Antioquia. Boletín.

EPIDEMIOLÓGICO DE ANTIOQUIA. No. 19 (oct. nov. 1994); p. 342-380

FAUCI, A. The AIDS epidemic. Considerations for the 21th Century. En: The New England Journal of Medi-cine. Vol.14, No. 341. (1999); p. 1046 -1050.

ORGANIZACIÓN PANAMERICANA DE LA SALUD. Situación de los bancos de sangre en la región de la Américas 1994 - 1995. Vol. 18, No.1 (1997) 
